\title{
Evaluation of water availability ability based on Dimensional Dynamic Fuzzy Comprehensive Evaluation Model
}

\author{
Jianchun Liu \\ School of North China Electric Power University, Baoding 071000, China; \\ 765999258@qq.com
}

Keywords: water availability ability, system indicators, coordination indicators, dynamic weights

\begin{abstract}
In this paper, Dimensional Dynamic Fuzzy Comprehensive Evaluation Model ${ }^{[1]}$ is devised to evaluate the water availability ability. Water availability can be understood by two main aspects: environment and economy. In the modeling process, the evaluation can be transformed into two-dimensional form. Dynamic modeling process adds time factor to obtain the different weights in different periods.
\end{abstract}

\section{Introduction}

Based on the two-dimensional modeling process, evaluation indicators should be selected in two aspects $^{[2]}$ : environmental system and comprehensive coordination. Environmental system reflects the regional water resource situation, which depends on the macro geographical environment, and can be defined as System Indicators (SI). While comprehensive coordination aspect should describe the relationships with economy, society and ecology systematically, which can be concluded as Coordination Indicators (CI). The summarized indicators are shown in Figure 1. The data for the indicators is collected from the websites ${ }^{[3][4]}$ mainly.

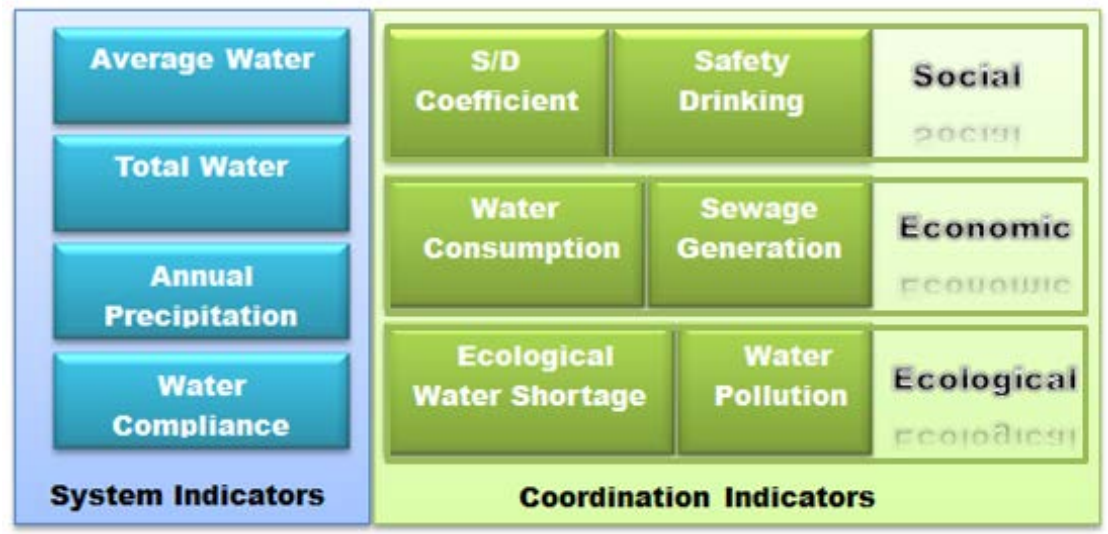

Figure 1 Water Availability Indicators

\section{System Indicators (SI)}

- Average Water. It measures the amount of freshwater resources can be utilized to the average share of each person, which approximates naturalized river discharge.

- Total Available Water (area normalized). Total available water is an estimate of surface water availability. It measures the ratio of average local available water to the area of this region.

- Annual Precipitation. It is a statistical data, which refers to the regional natural precipitation degree recharge.

- Water Compliance. This metrics is related to water quality to meet the requirements and average local water quality.

\section{Coordination Indicators (CI)}

Coordination Indicators (CI) can be subdivided into three aspects: Social Coordination Indicators, 
Economic Coordination Indicators and Ecological Coordination Indicators.

\section{Social Coordination Indicators:}

- S/D Coefficient. Literally, this metrics can be described as the ratio of water demand in the region to water supply.

- Safety Drinking. It refers to the proportion of people drinking safety water.

\section{Economic Coordination Indicators:}

- Water Consumption. It refers to the ratio of the total water consumption to the city's gross domestic product (GDP).

- Sewage Generation. It describes the ratio of total sewage generation to total GDP, which reflects the coordination of regional economic development and water pollution.

\section{Ecological Coordination Indicators:}

- Ecological Water Shortage. It measures the proportion of ecological environment in water scarce situation.

- Water Pollution. This metric refers to the coefficient of water pollution proportion.

\section{Dynamic Weights and Normalization}

\section{- Determine Dynamic Weights}

Coefficient of Variation Method ${ }^{[5]}$ is an objective weighting method, which establishes the objective weight of indicators, using the information contained in the indicators directly. Considering the dynamic nature of the indicators that affect both supply and demand, the developed model is devised based on the previous theory.

We specify the calculation of System Indicators (SI), and the others can be calculated in the same way. In the example, the selected countries are respectively China, the United States, Canada and Egypt.

The entire calculation flow is shown as follow:

Step 1: Construct the basic evaluation dataset $A$. Define the evaluation indicators index as $X_{i}(i=1,2,3,4)$ and the countries as $Y_{j}(j=1,2,3,4)$.

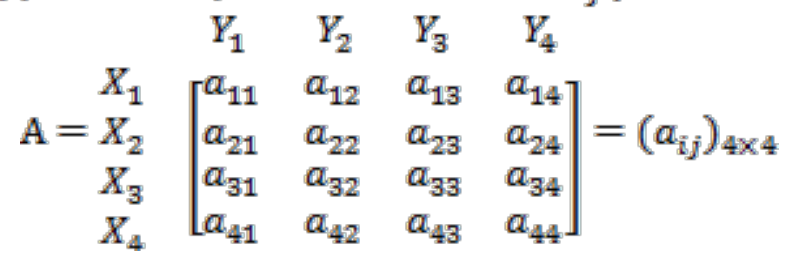

Step 2: Calculate the growth matrix $B$ considering the variation trends of indicators.

$$
\mathrm{B}=b_{i j}=\left\{\begin{array}{cl}
0 & i=1 \\
\frac{a_{i j}-a_{(i-1) j}}{\left|a_{(i-1) j}\right|} & i=2,3,4
\end{array}\right.
$$

where $b_{i j}$ describes the growth degrees of $a_{i j}$ compared with the previous data.

Step 3: Weighted summation. Integrate the static composite matrix $A$ with the growth matrix $B$ in the consideration of static evaluation and dynamic growth. The dynamic comprehensive evaluation matrix $C$ can be calculated as follow:

$$
\mathrm{C}=\left(c_{i j}\right)_{4 \times 4}=(1-s) \cdot a_{i j}+s \times b_{i j}, \quad i=1,2,3,4 ; j=1,2,3,4
$$

where $s$ is defined as time factor, a new indicator we introduce to the model, which reflects the different attentions to different period in the analysis process. The closer $s$ to 0 , the greater attention to recent evaluation data. Similarly, the closer $s$ to 1 , the changed evaluation data gets greater attention. In this example, we define $\mathbf{s}=0.5$, which presents static integrated value is as important as the growth value. 
$c_{i}=\frac{1}{4} \sum_{j=1}^{4} c_{i j}, \quad i=1,2,3,4$

Step 4: Calculate the average value $c_{\bar{i}}$ and standard deviation $S_{i}$ as follow:

$$
S_{i}=\sqrt{\frac{1}{4} \sum_{j=1}^{4}\left(c_{i j}-c_{j}\right)^{2}}, \quad i=1,2,3,4
$$

The coefficient of variation $V_{j}$ of indicators: $V_{j}=\frac{s_{i}}{c_{j}}, j=1,2,3,4$

$$
\omega_{j}=\frac{V_{j}}{\sum_{j=1}^{4} V_{j}}
$$

Obtain the weights of indicators after normalizing the coefficient of variation:

The calculated weights of System Indicators are shown in Table 1:

\begin{tabular}{|c|c|c|c|c|}
\hline & $\begin{array}{l}\text { Average } \\
\text { Water }\end{array}$ & $\begin{array}{l}\text { Available } \\
\text { Water }\end{array}$ & $\begin{array}{c}\text { Annual } \\
\text { Precipitation }\end{array}$ & $\begin{array}{c}\text { Water } \\
\text { Compliance }\end{array}$ \\
\hline Weight & 0.4545 & 0.1775 & 0.3026 & 0.0655 \\
\hline
\end{tabular}

Table 1 The Weights of System Indicators

- Normalization

Normalization process aims to determine membership functions. A fuzzy set is defined in terms of a membership function which maps the domain of System Indicators (SI) onto the interval [0,1]. The membership functions represent the degree that specified value belongs to the set.

Define $a_{i j}$ as the $a_{j}$ value for $i^{\text {th }}$ country and $\max _{j}\left(a_{i j}\right)$ as the maximum $a_{j}$ value for all indicators.

In the following equations, $r_{i j}$ is the proximity of $a_{i j}$ towards the relatively best value of indicators $i$ among all samples. For value of positive indicators, bigger is better, just as the following equation shown:

$$
r_{i j}=\frac{a_{i j}}{\max _{j}\left(a_{i j}\right)}
$$

Oppositely, as for absolute value of negative indicators, smaller is better:

$$
r_{i j}=\frac{\min _{j}\left(a_{i j}\right)}{a_{i j}}
$$

\section{Conclusion}

As an illustration to show the model's performance, the four countries (China, The United States, Canada and Egypt) continue to be used in the following implementation. We evaluate the four

\begin{tabular}{|c|c|c|c|c|}
\hline & China & The United States & Canada & Egypt \\
\hline System Indicators (SI) & 0.5133 & 0.8605 & 0.9790 & 0.1767 \\
\hline Coordination Indicators (CI) & 0.3559 & 0.6985 & 0.8860 & 0.1700 \\
\hline
\end{tabular}
countries from 2010 to 2014, and the results of all indicators divided into two categories are demonstrated in Table 2:

Table 2 The Scores of Indicators Sets for Countries

With SI value $y$ and CI value $x$ of a country in any given year, we can determine a position $(x, y)$ in two-dimensional coordinates plane just as shown in Figure 2. The distance between (x,y) and the origin of coordinates refers to the score of a country's ability to provide clean water in one year. The higher the score is, the more sustainable a country is. 


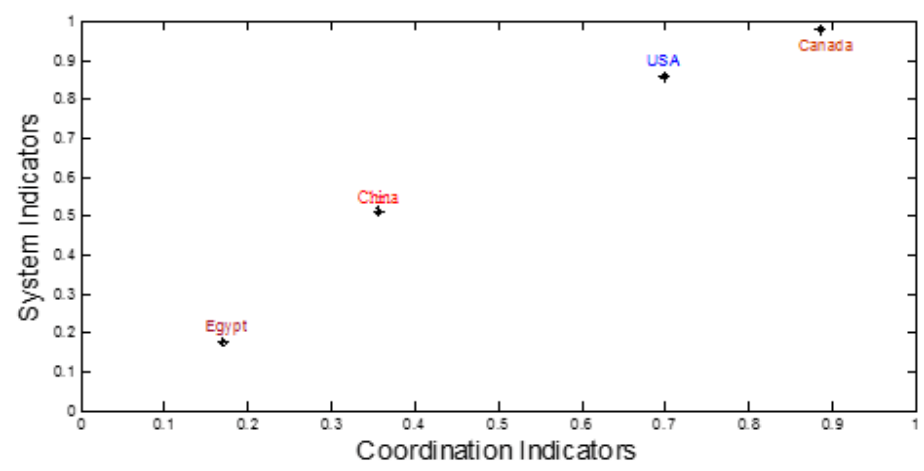

Figure 2 The Scores of Counties

\section{References}

[1]http://wenku.baidu.com/link?url=8TQEAgTPDm9sRb-sJt-d7CBtivKaHn6NVApTwKabFGjjU

[2]Jiajun Liu. Study on the Carrying Capacity of Water Resources in China[J].Journal of Natural Resource,2011,26(2): 261-270

[3]The World's Water: Information on the World's Freshwater Resources. (http://worldwater.org).

[4]GrowingBlue: Water. Economics. Life. (http://growingblue.com).

[5]https://en.wikipedia.org/wiki/Coefficient_of_determination 